\section{GOBERNANZA URBANA: REFLEXIONES A PARTIR \\ DE LOS DISTRITOS ECONÓMICOS DE LA CIUDAD DE BUENOS AIRES ${ }^{1}$}

Resumen

El artículo analiza la implementación de los distritos económicos en el sur de la ciudad de Buenos Aires. Desde un abordaje cualitativo, estudia las modalidades de intervención pública orientadas a la creación del "clima de negocios", incluyendo los beneficios impositivos, las inversiones en infraestructura, transporte, espacio urbano y equipamiento y el entramado discursivo puesto en juego para transformar las imágenes y sentidos que pesan sobre estas áreas de la ciudad. El artículo propone un cruce metodológico para dar cuenta de las formas específicas que asume el accionar público en

\section{URBAN GOVERNANCE: REFLECTIONS FROM THE ECONOMIC DISTRICTS OF THE CITY OF BUENOS AIRES}

Carolina Gonzalez Redondo

\section{Abstract}

The article analyses the implementation of the economic districts in the south of the city of Buenos Aires. From a qualitative approach, it studies the different components of public intervention aimed to create the "business environment", including tax benefits, investments in infrastructure, transportation, urban space and equipment; and the discursive framework put into play to transform the images and senses related to these areas of the city. The paper proposes a methodological crossover to take into account the specific forms that public action assumes in the public-private articulation. In this way, it 
la articulación público-privada. De este modo, permite dar contenido a categorías teóricas más abstractas, como empresarialismo urbano y neoliberalismo realmente existente, y a la vez reflexionar sobre los procesos de gobernanza urbana.

PALABRAS CLAVE: GOBERNANZA URBANA, EMPRESARIALISMO URBANO, CLUSTERS, RENOVACIÓN URBANA, ANÁLISIS DEL DISCURSO.

Recibido: 2020-04-13

Aceptado: 2020-08-25 allows to give content to more abstract theoretical categories, such as urban entrepreneurialism and existing neoliberalism, and at the same time to reflect on urban governance processes.

KEYWORDS: URBAN GOVERNANCE, URBAN ENTREPRENEURIALISM， CLUSTERS， URBAN RENEWAL, DISCOURSE ANALYSIS.

Received: 2020-04-13

Accepted: 2020-08-25

2 Becaria posdoctoral CONICET Instituto de Estudios de América Latina y el Caribe (IEALC), Facultad de Ciencias Sociales, Universidad de Buenos Aires, Argentina, http://orcid.org/0000-0002-8338-8148. Correo electrónico: carolina.gredondo@gmail.com 


\section{Introducción}

Desde 2008, el Gobierno de la Ciudad de Buenos Aires (GCBA) promueve la radicación de empresas e instituciones de un mismo sector económico en áreas específicas de la ciudad, denominadas distritos económicos. Para la atracción de estos emprendimientos, el gobierno local ofrece incentivos tributarios, financieros e inmobiliarios, despliega estrategias para la articulación con los actores empresariales promovidos y realiza una fuerte inversión pública orientada a la renovación urbana de estas áreas. Estos distritos conforman una política paraguas que profundiza y extiende el proceso de renovación urbana desplegado en el sur de la ciudad desde la década del '90. En sintonía con los discursos sobre gobernanza urbana (CEPAL, 2018; "Cities in transition", 2000; "Nueva agenda urbana", 2017; OECD, 2000), y retomando premisas del modelo de cluster (Porter, 1995), la política de distritos se apoya en la "sinergia público-privada" para fomentar el desarrollo económico, urbano y social de estas "zonas deterioradas" de la ciudad.

Reconociendo las fronteras lábiles y porosas entre el Estado y el ámbito privado (Bohoslavsky y Soprano, 2008; Marques, 2019; Menazzi y Jajamovich, 2019; Vommaro y Gené, 2017), en este artículo nos preguntamos ¿en qué consiste la "sinergia público-privada" que promueve la política de distritos?; ¿cómo se produce y qué vínculos expresan dicha "sinergia"?; ¿qué tipo de procesos y dinámicas desencadenan en el territorio? Para responder estas interrogantes, analizamos la implementación de la política de distritos, atendiendo a las modalidades de intervención pública orientadas a la creación del "clima de negocios" necesario para atraer inversiones a estos barrios de la ciudad.

Desde un abordaje cualitativo, analizamos los diversos componentes de la intervención pública, desde los beneficios impositivos -propios de la política de distritos- hasta las inversiones en infraestructura, transporte, espacio urbano y equipamiento, así como también los aspectos simbólicos de la política. Esto supone considerar el entramado discursivo que ponen en juego los distintos actores para transformar las imágenes y sentidos que pesan sobre estas áreas de la ciudad. Así, elementos que podrían pensarse como iniciativas aisladas, aquí son analizados en su totalidad, teniendo en cuenta cómo se solapan y complementan las distintas intervenciones sobre el territorio.

El artículo propone un cruce metodológico para analizar en forma complementaria las distintas dimensiones del accionar público. Asimismo, resalta la importancia de considerar las dimensiones espacial y temporal en el estudio de los procesos y políticas urbanas, sobre todo para dar cuenta de sus continuidades y persistencias. En tercer término, enfatiza la capacidad de agencia de los actores y sus trayectorias en el devenir de una política urbana. En este sentido, la inclusión de 
herramientas provistas por la sociología política invita a pensar al Estado en su heterogeneidad. Por último, el artículo da cuenta de las formas específicas que asume el accionar público en la articulación público-privada. De este modo, se advierten las particularidades que asume el empresarialismo urbano (Harvey, 1989) y el neoliberalismo realmente existente (Theodore, Peck, y Brenner, 2009) en la ciudad de Buenos Aires y se reflexiona sobre los alcances y limitaciones de la gobernanza urbana.

\section{Recorte espacio-temporal}

Si bien existen cinco distritos económicos (Figura 1), en este artículo, focalizamos en los tres ubicados en la Comuna 4: artes, diseño y tecnológico. Dado que nuestro foco es el sur de la ciudad, el distrito audiovisual -ubicado en los barrios del norte, con características territoriales muy diferentes- no forma parte de nuestro análisis. Asimismo, dejamos de lado el distrito del deporte, situado en la comuna 8, porque la intervención estatal en esta zona adopta características específicas que lo diferencian de los otros casos de estudio (Arqueros Mejica, 2019).

En 2008 se crea el distrito tecnológico, que promueve la radicación de empresas vinculadas a las Tecnologías de la Información y la Comunicación (TIC) en un polígono que abarca Parque Patricios, y un sector de Nueva Pompeya y Boedo. A fines de 2012, se sanciona la ley del distrito de las artes que busca la instalación de emprendimientos culturales en el barrio de La Boca y el sector sur de San Telmo. En el distrito del diseño, creado en 2013 en el barrio de Barracas, se incentiva la instalación de firmas dedicadas al diseño en distintos rubros.

\section{Marco teórico y antecedentes}

La política de distritos retoma elementos de un modelo de gestión urbana de gran difusión, como es el cluster (Porter, 1995, 1998)3. El supuesto central de la noción de cluster es que la proximidad geográfica entre empresas e instituciones de un mismo sector económico generaintercambiosquefavorecen el crecimiento de toda la cadena, a través de las denominadas "sinergias". El otro supuesto es que los clusters motorizan procesos de desarrollo en la medida

3 Este modelo, que hegemonizó las agendas de investigación y de política pública, retoma varios de los principios en que se sustentan las políticas de fomento a las economías de aglomeración, desarrolladas principalmente por la geografía económica (Fernández, Amin y Vigil, 2008). Aunque Porter no lo cite explícitamente, en sus desarrollos se reconocen varios de los principios en los que se sustenta el concepto de distrito industrial, acuñado por Alfred Marshall y reelaborado por Becattini (2002). En su entramado discursivo, la política de distritos combina la noción de cluster con elementos de estos otros modelos, así como también con algunas propuestas sobre economías y ciudades creativas. Por cuestiones de espacio no podemos profundizar en esto, sugerimos Gonzalez Redondo, 2019. 
FIGURA 1. MAPA DE LOS DISTRITOS DE LA CIUDAD DE BUENOS AIRES.

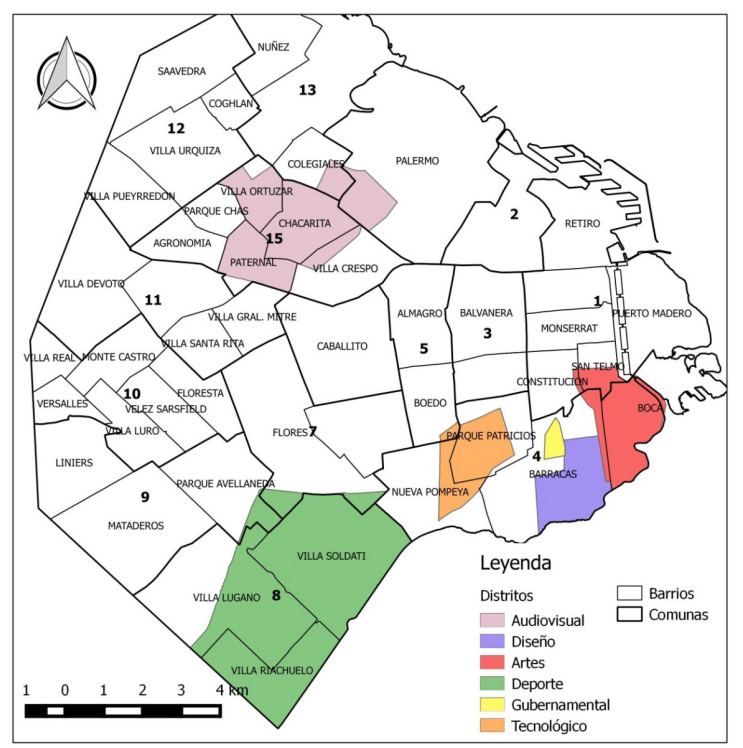

Fuente: elaboración propia en base a información del GCBA

en que generan actividad económica, que derrama en inversiones y empleo (Porter, 1995, 1998). Según este modelo, el Estado debe pasar de un rol de provisión directa a uno de facilitador, identificando y potenciando las ventajas competitivas de su territorio que colaboran en la gestación del "clima de negocios", que es lo que permite las mejoras en la productividad de las empresas (Porter, 1995, 1998).

Este rol facilitador del Estado se articula conceptualmente con los enfoques sobre gobernanza que cobran fuerza durante los
$90^{4}$ y que, una década más tarde, se trasladan al plano de la gestión local, dando lugar al término gobernanza urbana. Este concepto de carácter normativo (Aguilar, 2016) establece las bases para el buen gobierno de las ciudades, en el marco de los nuevos desafíos impuestos por la economía global. Uno de sus argumentos principales es que la complejidad de los problemas urbanos actuales dificulta su abordaje por parte del gobierno local, haciendo imprescindible la articulación con otros actores no gubernamentales ("Cities in transition", 2000; CEPAL, 2018; "Nueva agenda urbana", 2017; OECD, 2000; Salmerón Escobar, 2017;). El discurso sobre buena gobernanza urbana fue muy influyente en América Latina, lo que se manifestó en la privatización de servicios públicos, la incorporación de instrumentos de normativa urbana orientados a la promoción de la articulación público-privada, y la proliferación de planes estratégicos elaborados bajo la tutela de consultores internacionales (Dávila, 2014; Pírez, 2016; Zurbriggen, 2011). Casi dos décadas más tarde, sus lineamientos continúan vigentes en documentos más recientes (CEPAL, 2018; "Nueva agenda urbana", 2017).

$4 \quad$ Estos enfoques surgen en Europa occidental, en el marco de las preocupaciones sobre la crisis del Estado de Bienestar. Este término se desarrolla inicialmente como un concepto descriptivo con múltiples acepciones (Aguilar, 2016) que, en su proceso de circulación, se transforma en un concepto normativo, que señala el tipo de gobierno "apropiado" para el nuevo milenio (Aguilar, 2016; Marques, 2013). 
Desde estas perspectivas, la gestión urbana y la ciudad se conforman como un sujeto económico que adopta una lógica empresarial y mercantil (Vainer, 2000). A este tipo de gestión urbana, caracterizada por la preeminencia de una visión empresarial sobre la ciudad y sobre lo público, Harvey (1989) la conceptualiza como empresarialismo urbano. Este concepto refiere a un tipo de gestión que "descansa más en una asociación público-privada focalizada en inversión y desarrollo económico con una construcción especulativa del lugar, que en el mejoramiento de las condiciones del territorio" (Harvey, 1989, p. 89). Aquí el Estado adopta un rol de facilitador y promotor de la actividad económica, asumiendo los riesgos y garantizando la rentabilidad del capital privado.

Retomando algunos de estos elementos, en este artículo analizamos bajo qué supuestos, mediante qué herramientas y modalidades se produce la articulación entre actores estatales y empresariales en la implementación de la política de distritos. Nos centramos en las modalidades de intervención pública, dejando para futuros trabajos el análisis de las respuestas que producen los actores empresariales a los incentivos públicos. Esto nos permite reflexionar sobre los alcances y limitaciones de los procesos de gobernanza urbana y, a la vez, repensar algunas categorías como empresarialismo urbano (Harvey, 1989) y neoliberalismo realmente existente (Theodore et al., 2009). Para esto último resulta indispensable desagregar el Estado y analizarlo en su heterogeneidad, reconociendo los múltiples intereses y contradicciones que permean el accionar público.

El análisis de políticas públicas requiere considerar varios elementos: los actores involucrados en su formulación y procesamiento; los marcos institucionales y socioculturales en los que éstos transitan; las reglas implícitas que orientan sus acciones y comportamientos, y las narrativas que contribuyen a su legitimación (Shore, 2010). En este artículo intentamos dar cuenta de estos múltiples aspectos, teniendo en cuenta a los actores estatales y sus trayectorias biográficas y a las narrativas que se ponen en juego en el entramado discursivo de la política.

En este sentido, cabe sostener que, en primer lugar, las políticas públicas son producidas a partir de la interacción desigual de diversos actores (Marques, 2013). Aquí focalizamos en los actores estatales, concebidos como todos aquellos organismos de los distintos niveles gobierno -junto con los funcionarios y agentes públicos que en ellos transitan-involucrados en la implementación de esta política. Incorporando herramientas de la sociología política (Bohoslavsky y Soprano, 2008; Gené, 2011; Giorgi, 2014; Vommaro y Gené, 2017), atendemos a las trayectorias biográficas y profesionales de los sujetos que habitan y dan vida a la política de distritos (Bohoslavsky y Soprano, 2008), abordaje también sugerido por la antropología de las políticas públicas (Shore, 2010). 
En segundo lugar, destacamos la complejidad de las políticas urbanas debido al carácter territorializado del espacio (Lopes de Souza, 1995; Santos, 2000), cuando la política se encuentra con el territorio, las particularidades de este último redefinen su curso de acción (Rodríguez y Di Virgilio, 2011). Concebimos a las políticas urbanas como procesos sociopolíticos conflictivos en los que intervienen actores con intereses y concepciones sobre lo urbano en disputa (Di Virgilio y Rodríguez, 2016). Son precisamente estas relaciones desiguales de poder entre los diversos actores las que se omiten en los enfoques más edulcorados sobre gobernanza urbana, lo que termina favoreciendo los intereses de aquellos actores con mayor peso y capacidad para transformar la ciudad (Mattos, 2008).

\section{Consideraciones metodológicas}

Desplegamos una estrategia cualitativa, con orientación empírica e interpretativa. Para la producción de evidencia empírica, combinamos diversas fuentes y técnicas, como documentos elaborados por el GCBA (marcos normativos, partidas presupuestarias, gacetillas de prensa y materiales gráficos); y entrevistas semi-estructuradas a actores involucrados en la política (funcionarios públicos, agentes del GCBA, empresarios, agentes culturales) 5 . Combinamos un abordaje interpretativo con el trabajo con sistemas de información geográfica (SIG) y con un dispositivo para el análisis de los discursos. En el primer caso, recuperamos información elaborada por el GCBA (planes plurianuales de inversión, inversiones en obras públicas, información sobre transporte, infraestructura y equipamiento urbano $^{6}$ ) y la procesamos mediante SIG. El análisis de información georreferenciada permitió advertir cómo se distribuyen diferencialmente las inversiones públicas en los tres distritos.

Luego, a través del análisis discursivo abordamos la dimensión simbólica de la política: identificamos los roles que asume el gobierno local en la ejecución de esta política y las narrativas que se construyen en torno a los barrios que son objeto de intervención. Construimos un dispositivo que, retomando la perspectiva teórica de la escuela francesa, combina elementos de las teorías de la enunciación y la argumentación ${ }^{7}$. Particularmente, el análisis de

5 Se realizaron 29 entrevistas entre 2016 y 2019. Asimismo, incorporamos siete entrevistas realizadas por colegas en el marco de sus investigaciones, a quienes agradecemos especialmente por compartirlas: Eugenia Goicoechea, Ivana Socoloff y Natalia Lerena.

6 Esta información se encuentra disponible en "Buenos Aires Data", s.f.

$7 \quad$ Esta perspectiva invita a pensar la dimensión conflictiva de todo discurso en tanto que su sentido no es unívoco, existe una lucha por su fijación. No hay una "democracia 
las estrategias argumentativas ayuda a identificar las construcciones, tematizaciones y narrativas puestas en juego en el entramado discursivo de la política.

\section{Resultados}

\section{ESTADO FACILITADOR Y MEDIADOR}

En este apartado, profundizamos en el trabajo cotidiano de los equipos de los distritos, las estrategias y vínculos que establecen con el sector privado. El Estado local asume un rol activo en la creación del "clima de negocios", generando oportunidades de negocio con el fin de atraer nuevos inversores a las áreas promocionadas. En este hacer, el Estado local no sólo asume un papel de facilitador, identificando las "ventajas" del territorio y generando las condiciones para una inversión segura, sino que además se transforma en el mediador entre los distintos actores involucrados en la política. Veamos en qué consisten estos roles de facilitador y mediador.

Desde los orígenes de los distritos, el GCBA crea una estructura orientada a la atracción de inversiones, que fue cambiando a lo largo del tiempo y de las distintas gestiones de gobierno. Identificamos tres etapas. La primera

discursiva", las tematizaciones no son aleatorias, sino que responden a tendencias hegemónicas que regulan lo posible de ser dicho en un momento dado (Angenot, 2010). corresponde a las dos gestiones de Mauricio Macri como jefe de gobierno (2007-2015), en la que los distritos estuvieron a cargo de las áreas de Inversiones y de Economías Creativas dentro del Ministerio de Desarrollo Económico (MDE), con funcionarios - de amplia trayectoria en el mundo empresarial- que cumplieron un rol central en el diseño de la política: Francisco Cabrera, Carlos Pirovano y, posteriormente, Enrique Avogadro. En diciembre de 2015, Mauricio Macri asumió como presidente de la Nación y muchos de estos funcionarios pasaron a la órbita nacional. En la ciudad de Buenos Aires, el nuevo jefe de gobierno Horacio Rodríguez Larreta, inauguró una nueva gestión del mismo signo político. El MDE se transformó en Ministerio de Modernización, Innovación y Tecnología, y quedó a cargo de Andy Freire, quien le dio una nueva impronta al ministerio y a los distritos económicos. En esta segunda etapa (2016-2017) la gestión de los distritos se vio parcialmente interrumpida, debido a los cambios en la estructura ministerial en todos los niveles (coordinaciones, gerencias, direcciones y subsecretarías).

A partir de 2018, los distritos que quedan a cargo de la Dirección General (DG) de Distritos Económicos dentro del renovado Ministerio de Economía y Finanzas. Esta DG tiene a su vez una Gerencia Operativa, compuesta por una coordinación de cada distrito, con sus respectivos agentes. El DT tiene una mayor estructura (con cuatro agentes y un 
coordinador), mientras que los distritos del diseño y de las artes sólo tienen un coordinador y un agente cada uno (a enero de 2019) ${ }^{8}$. Estos equipos son la "veta más comercial de los distritos" (entrevista a la Gerente Operativa de los Distritos Económicos, enero de 2019), se encargan de la atracción de empresas e inversiones y acompañan a los beneficiarios antes, durante y luego de su radicación. Si bien la creación de esta DG de Distritos Económicos implicó una mayor estabilidad institucional, los beneficiarios entrevistados advierten en todos los casos que su rol no "volvió a ser el mismo", ya que los distritos no retomaron el impulso previo al cambio de gestión de $2015^{9}$.

Actualmente, la DG de Distritos Económicos se ocupa del sostenimiento cotidiano de la política. $\mathrm{Su}$ director es Juan Manuel Seco, un joven licenciado en Contabilidad y Administración de Empresas por la UADE, con un Master en Administración de Empresas en el Instituto de Tecnología de Massachusetts, que desarrolló

8 Posteriormente, hubo nuevos cambios en la estructura institucional, que expresan la pérdida de jerarquía y de peso de los distritos en la agenda del gobierno local.

9 La pérdida de peso de los distritos económicos dentro de la agenda pública local se relaciona con las reconfiguraciones del escenario político nacional. La convergencia de un mismo signo político en el Gobierno Nacional, la Ciudad y la Provincia de Buenos Aires habilitó una serie de intervenciones en materia urbana (la urbanización de villas, la mercantilización de inmuebles y terrenos públicos y la sanción de un nuevo Código Urbano) que reorganizaron las prioridades de la gestión local. Para profundizar ver Gonzalez Redondo, 2019. su carrera en el exterior, mayormente en las áreas financieras de grandes compañías, hasta su desembarco en la gestión pública. Lo sucede como Gerente Operativa (GO) de los Distritos, Florencia Cambré, licenciada en Marketing por la Universidad del CAECE, quien trabaja en el GCBA desde $2012^{10}$. Los coordinadores de cada distrito son en general jóvenes profesionales, que ingresaron al GCBA entre los años 2012 y 2016 y pasaron por distintos cargos dentro del área, mostrando en todos los casos una movilidad ascendente dentro de la estructura institucional. En sus discursos y prácticas, estos agentes se alejan del perfil técnico asociado al saber experto en un campo disciplinario y, en cambio, se presentan como agentes dinámicos y pragmáticos que ponen en juego un saber generalista orientado a "mover procesos" (Vommaro, 2017). Incorporando saberes, destrezas, éticas y estéticas del mundo privado, estos agentes encaran su objetivo principal: la atracción de inversiones a las áreas de los distritos. Para ello, negocian con cámaras empresariales, organizan recorridos -para que los potenciales beneficiarios conozcan los distritos- y facilitan todo el proceso de radicación, asesorando a las empresas en la inscripción al registro de beneficiarios y en la búsqueda de un espacio para instalarse.

10 Florencia Cambré fue coordinadora del Distrito Tecnológico entre 2012 y 2016, momento en que asume como GO tomando a su cargo la gestión de todos los distritos. 
En su estrategia de acompañamiento a las empresas, las coordinaciones de los distritos actúan como mediadores entre los potenciales beneficiarios y los agentes inmobiliarios; pero también entre estas firmas y distintos organismos públicos locales y nacionales. Así, los referentes distritales asumen las tareas de un gestor; así, "ayudando a acelerar los procesos" (entrevista a la Gerente Operativa de los Distritos Económicos, enero de 2019.), hacen el seguimiento del trámite de inscripción al registro de beneficiarios, negociando con las agencias recaudatorias y facilitando otro tipo de gestiones ante diferentes entes de gobierno (habilitaciones de establecimientos, permisos de obra, etc.). Al tornarse referentes, estos equipos concentran las distintas demandas de los privados al Estado.

Este rol de las coordinaciones de los distritos como facilitadores y mediadores es valorado positivamente por la mayoría de los beneficiarios entrevistados. En sus discursos prima cierta sorpresa al reconocer que su accionar se distancia del imaginario más extendido sobre el Estado y sus prácticas burocráticas. Así, los equipos de los distritos y sus referentes condensan una serie de representaciones asociadas a las prácticas del sector privado (con lexemas como "moderno", "ágil", "simple", "ameno") que los diferencia de las otras agencias públicas y sus empleados burócratas, agrupados en torno a la figura de "la Muni"11. Es recurrente en los discursos de estos empresarios la noción de un distrito "con cara"12, siempre disponible y atento a sus necesidades, que responde a sus demandas (o al menos lo intenta) y facilita sus gestiones, frente a las trabas que supone el accionar de "la vieja Municipalidad".

Sin embargo, este Estado facilitador y mediador expresa sus limitaciones en las disputas entre las distintas agencias, ya sea "luchando" contra las trabas burocráticas de "la Muni", o negociando a partir de los distintos objetivos de estos organismos. Mientras que la coordinación de los distritos tiene entre sus metas la radicación de empresas, la agencia recaudatoria tiene como objetivo el aumento de la recaudación. Estas distintas prioridades se expresan en tensiones intra-estatales que inciden en el derrotero de la política, recordándonos que el Estado es un espacio polifónico (Bohoslavsky y Soprano, 2008).

Este rol mediador constituye una estrategia política de la nueva gestión local (desde 2007), -no exclusiva de esta política- para alterar el tejido relacional del Estado (Marques, 2019),

11 Refiere a la "vieja Municipalidad" de la ciudad de Buenos Aires que, a partir de su autonomización en 1996, cambió por el Gobierno de la Ciudad de Buenos Aires. Las prácticas burocráticas siguen asociándose a distintos organismos sedimentados desde la etapa de la Municipalidad.

12 "(...) la verdad es que es un vínculo muy ágil, muy presente. Siempre tiene cara el distrito. Desde el día uno." (Entrevista a Mariana, empresaria del DT, marzo de 2019). 
entendido como aquella estructura relacional relativamente estable, de carácter fuertemente inercial, que construye permanencias, estructura influencias, alianzas y oposiciones políticas, organizando la permeabilidad del Estado a las demandas de los distintos actores sociales y empresariales (Marques, 2019, p. 7). La "vieja Municipalidad" -con sus continuidades y permanencias- puede asociarse a ese tejido relacional del Estado que la gestión local busca alterar, incorporando una estructura institucional con agentes de un perfil "dinámico" y "moderno", dotados de un "bilingüismo" que les permite comprender cómo piensan los empresarios, habilitando de este modo un diálogo más fluido entre actores estatales y empresariales (Vommaro, 2017). De hecho, las modalidades de intervención de las coordinaciones de los distritos son interpretadas por los empresarios en el marco de un lenguaje compartido, en el que el gobierno local aparece hablando el "mismo idioma", desplegando repertorios de acción y visiones del mundo más afines a las suyas y que se diferencian de las modalidades que reconocen como características del sector público.

Ahora bien, este Estado facilitador y mediador no interviene con la misma intensidad en todos los distritos ni con todos los actores. Si bien los equipos de los distritos son el interlocutor de empresas, desarrolladores y agentes culturales con otras áreas de gobierno para resolver los problemas cotidianos, su respuesta es diferente según los distintos actores. Especialmente cumple un rol activo en la creación del "clima de negocios", facilitando las oportunidades de negocios, lo que requiere mediar entre los distintos actores involucrados en la política. Un rol que consiste en asegurar la rentabilidad del sector privado, tanto desde la oferta como desde la demanda: no sólo se garantiza a empresas e inversores mejores márgenes de rentabilidad a través de las exenciones impositivas y la movilización de suelo, sino que además se busca generar "oportunidades de negocio", promoviendo vínculos entre los distintos agentes del sector privado (Gonzalez Redondo, 2019).

Esta facilitación de los negocios no exige ninguna contraprestación por parte de los actores empresariales, quienes actúan librados a su "buena voluntad". En este sentido, si bien podemos hablar de un tipo de gestión enmarcada en lo que Harvey denomina empresarialismo urbano, ésta cobra formas diferentes a las que se generaron en las ciudades estadounidenses a partir de los años '70, donde fue acuñado el término. Mientras que allí los gobiernos locales imitaron prácticas de financiamiento del sector privado y asumieron una actitud empresarial que incluye la captura (al menos parcial) de la inversión pública (Leitner, 1990); en el caso de los distritos porteños la inversión pública no exige requisitos ni contraprestaciones. Parafraseando a Vainer (2000), se configura la ciudad como mercancía, como objeto pasivo para ser vendido y consumido; aunque la ciudad 
como empresa se queda a medio camino, pues sólo retoma de ésta sus estrategias y argumentos de venta, pero no su capacidad para recuperar la inversión. Como reconoce un funcionario, "el Estado interviniendo estratégicamente genera valor, modifica las condiciones de un determinado sector y crea nuevas oportunidades" (intervención del ex Ministro de Desarrollo Urbano en la Expo Real Estate de 2011). Así, el Estado realiza la mayor parte de la inversión y asume los riesgos, mientras que el privado capitaliza la plusvalía generada por la intervención pública. Como veremos, "el resultado en términos urbanísticos es por demás deficitario, porque el capital privado actúa de manera individual y fragmentada, poniendo de relieve las contradicciones de la urbanización capitalista" (Di Virgilio y Guevara, 2014, p. 14).

\section{Renovación urbana en los barrios de los distritos}

\section{DIMENSIÓN MATERIAL: ANÁLISIS DE LA INVERSIÓN PÚBLICA}

Contrariamente a la idea de que en los distritos "la inversión del Estado fue mínima" (entrevista a agente del distrito tecnológico, diciembre de 2018), la creación del "clima de negocios" se traduce en una fuerte inversión pública orientada a potenciar las "ventajas competitivas" de estas áreas. Distinguimos tres tipos de inversión pública en esta política: el costo fiscal derivado de las exenciones impositivas previstas en las leyes de cada distrito ${ }^{13}$; las partidas presupuestarias destinadas a las reparticiones encargadas de la ejecución de la política, y la inversión en obra pública en las áreas de intervención. Por restricciones de espacio, aquí sólo consideramos esta última, dejando las otras dos para futuros trabajos (Gonzalez Redondo, 2019).

Los incentivos fiscales y estrategias desplegadas por el gobierno local se refuerzan con iniciativas públicas de renovación urbana, que manifiestan el esfuerzo del Estado facilitador para convertir a estas zonas de la ciudad en sitios atractivos para la localización del capital. Observamos una fuerte inversión pública en infraestructura, transporte, seguridad y espacio público (Arqueros Mejica y Gonzalez Redondo, 2017; Goicoechea, 2016), que se despliega de manera desigual en el territorio, en articulación con los procesos previos de renovación de cada zona (Gonzalez Redondo, 2018).

La Figura 2 muestra las inversiones públicas realizadas en las áreas de los distritos de la Comuna 4 y su entorno, entre los años 2011

13 En términos brutos, el distrito tecnológico es por lejos el que insume un mayor costo fiscal $(\$ 5.998 .675 .126)$, seguido por el distrito del diseño (\$137.553.696) y, muy por debajo, el distrito de las artes (\$2.263744). Si analizamos los datos estimando las exenciones por beneficiario y por año, el DT representa un $55 \%$, seguido por el DDi con un $44 \%$ y casi una nula representación del DA, con un $1 \%$. Así, advertimos una distribución muy desigual del costo fiscal de esta política entre los tres distritos de la comuna 4. 
y 2019. Cabe señalar que no aparecen aquí algunas inversiones previas a 2011, como la puesta en valor de edificios patrimoniales destinados a usos culturales y las grandes sumas relacionadas con la extensión de la línea $\mathrm{H}$, que afectó directamente al distrito tecnológico. Dejando aparte estas dos cuestiones, la mayor inversión se concentra en el distrito de las artes y su entorno (56\%), destinada a obras hidráulicas, oficinas públicas (el reacondicionamiento del Edificio Lezama) y espacio público.

El distrito tecnológico y su entorno, en tanto, concentran el 34\% de la inversión, destinada en un $42 \%$ a obras de remodelación en centros de salud, lo que es predecible, dado que allí se congregan varios hospitales públicos. También se advierte una importante inversión en equipamiento educativo, un $15 \%$ en la puesta en valor de espacios verdes; y un 11\% a seguridad. Sin embargo, la mayor inversión es la extensión de la línea $\mathrm{H}$ del subte, que según los Planes Plurianuales de Inversión (PPI) (2008-2019) ronda los 107,2 mil millones de pesos. Si consideramos esta obra, el DT es por lejos el distrito que concentra la mayor inversión pública. El distrito del diseño y sus áreas cercanas reúnen únicamente un 10\% de la inversión total. La mayor parte está vinculada a obras de mantenimiento de los hospitales públicos de la zona aledaña. La inversión en oficinas públicas estuvo destinada a obras de refacción de la subsede de la Comuna 4; mientras que la de espacio público se destinó a la puesta en valor de la Av. Montes de Oca, principal arteria comercial de Barracas y zona más consolidada del distrito.

Una visualización de los tres distritos en conjunto (Figura 3) permite advertir cómo la inversión en obra pública se distribuye de manera diferencial entre e intra distritos. El DT concentra la mayor proporción, si consideramos la extensión de la línea $\mathrm{H}$; mientras que el distrito del diseño tiene la menor proporción. Dentro de cada distrito, las inversiones públicas se despliegan mayormente en los sectores más consolidados, potenciando los atributos de centralidad preexistentes en cada caso. Asimismo, se observan subsectores dentro de cada distrito en donde prácticamente no se han realizado inversiones: el extremo sudoeste del DDi (entre las vías del ferrocarril y la avenida Velez Sarfield) y el área sur de los distritos tecnológico y de las artes.

En el DT, la mayoría de las inversiones se concentran en las áreas ya consolidadas de Parque Patricios. Las obras de mayor impacto son aquellas dirigidas a mejorar los accesos y la conectividad del barrio, fundamentalmente la extensión de la línea $\mathrm{H}$ y, en menor medida, la creación del Metrobus Sur y el trazado de bicisendas. Otras inversiones importantes son la ampliación del cableado de fibra óptica (requisito solicitado por las empresas TIC) y de la red de wifi; la puesta en valor del Parque Patricios y de otros espacios verdes; 
y la instalación de las oficinas de la Jefatura del Gobierno de la Ciudad. A diferencia de lo que sucede en el distrito de las artes, todas las obras proyectadas fueron finalizadas y son valoradas positivamente por los empresarios y agentes inmobiliarios entrevistados. Asimismo, la distribución geográfica de estas inversiones coincide con la distribución de las nuevas empresas y emprendimientos inmobiliarios que se instalaron en el distrito.

En el distrito de las artes, las inversiones en obra pública se distribuyen en tres sectores: el área del Parque Lezama, el entorno de la Usina del Arte y el enclave de Caminito y Vuelta de Rocha (Figura 3). En el primer caso, las inversiones se concentraron en la puesta en valor del parque, la rehabilitación de la ex fábrica Canale para la instalación oficinas del GCBA y la refacción del Museo de Arte Moderno. En el segundo sector, la mayor inversión fue la refuncionalización de la Usina del Arte y las obras del bajo autopista (Dique Cero), que tienen como objetivo conectar Puerto Madero con Caminito. En Caminito, en tanto, las inversiones incluyen la puesta en valor de inmuebles patrimoniales y la creación de un paseo ribereño hacia el sur. Una obra con gran impacto en la conectividad del distrito es el Paseo de Bajo, inaugurado en 2019, que agiliza el acceso vehicular a esta zona de la ciudad.

En tanto, las inversiones en obra pública en el distrito del diseño son mucho menores tanto en envergadura como en los montos involucrados.
Estas obras se limitan a algunas intervenciones de maquillaje urbano sobre el eje más consolidado del barrio, la Av. Montes de Oca y el entorno del CMD (Figura 3). A diferencia de los otros casos, en el DDi no se hicieron (ni se proyectaron) inversiones en transporte.

\section{Dimensión simbólica: narrativas sobre los distritos}

Las intervenciones públicas se acompañan, como toda política de renovación urbana, de narrativas legitimadoras (Franquesa, 2007; Marcús y Vázquez, 2017; Stanchieri y Aricó, 2013) que -en tanto prácticas discursivas que agregan o quitan valor a un espacio determinado- matizan el peso económico de estos proyectos y a la vez los presentan como algo necesario y positivo (Franquesa, 2007, p. 128). A su vez, al intentar orientar y condicionar las prácticas espaciales de la ciudadanía (Thomasz, 2017), esta dimensión simbólica de la renovación urbana busca atraer nuevos visitantes, inversores y consumidores.

Esto se produce de manera diferencial en cada distrito, articulándose con las narrativas preexistentes sobre estos barrios y con los procesos de renovación previos. Las características de la actividad promovida y las inversiones desplegadas en cada distrito 
FIGURA 2. INVERSIÓN ACUMULADA EN OBRAS PÚBLICAS EN LAS ÁREAS DE LOS DISTRITOS Y ALREDEDORES (2011-2019), EN PESOS AJUSTADA A PRECIOS CONSTANTES DE DICIEMBRE DE 2018.

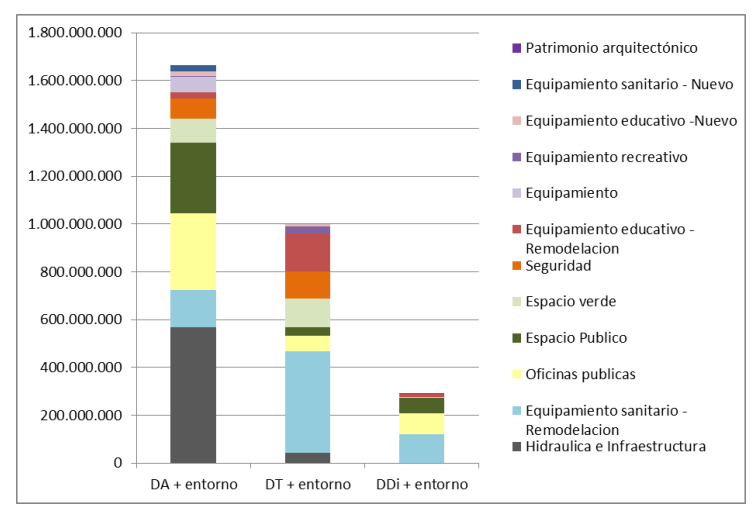

Fuente: Elaboración propia en base a BA Obras, s.f.

también influyen en las características que asume la dimensión simbólica de la renovación. Así, las mayores dificultades para atraer empresas e inversiones a los distritos de las artes y del diseño hicieron que, tanto en La Boca como en Barracas, la creación de los distritos se articulara con políticas de activación patrimonial y turistificación que buscaban atraer visitantes-consumidores (siguiendo el eje de las intervenciones precedentes). Para ello, el gobierno local busca revertir la carga simbólica de estas áreas "deterioradas" proponiendo nuevas narrativas sobre el lugar.
El distrito de las artes comprende dos barrios (San Telmo y La Boca) que son tematizados en los relatos oficiales como "históricos" y "emblemáticos". A San Telmo se lo reconoce por ser parte del casco histórico y por conservar gran parte de su patrimonio arquitectónico (Gonzalez Redondo, 2017). De La Boca se destaca por su origen portuario y por su caudal de inmigración europea, lo que lo torna "singular", "pintoresco" y "auténtico". Esta imagen hegemónicamente positiva de La Boca -ampliamente difundida- convive con otra que se construye como su reverso en base a aspectos negativos, vinculados a la presencia de sectores populares, su hacinamiento en conventillos y las representaciones vinculadas a la falta de higiene y la delincuencia (Thomasz, 2017). Mientras la positiva se elabora sobre el enclave turístico tradicional, la negativa se focaliza en espacios considerados "peligrosos" (Barrio Chino, Plaza Solís, Calle Nechochea, Bajoautopista).

En este distrito, la dimensión simbólica de la renovación busca revertir esta imagen negativa. Por tal motivo, las intervenciones se acompañan de un entramado discusivo que las legitima, un proceso de resemantización simbólico (Thomasz, 2017) que busca imponer un nuevo imaginario urbano que se nutre de las representaciones preexistentes, pero subvirtiéndolas, modificándolas y 
FIGURA 3. MAPA DE DISTRIBUCIÓN GEOGRÁFICA DE SERVICIOS Y EQUIPAMIENTOS, JUNTO CON LAS INVERSIONES EN OBRA PÚBLICA EN LOS TRES DISTRITOS DE LA COMUNA 4.

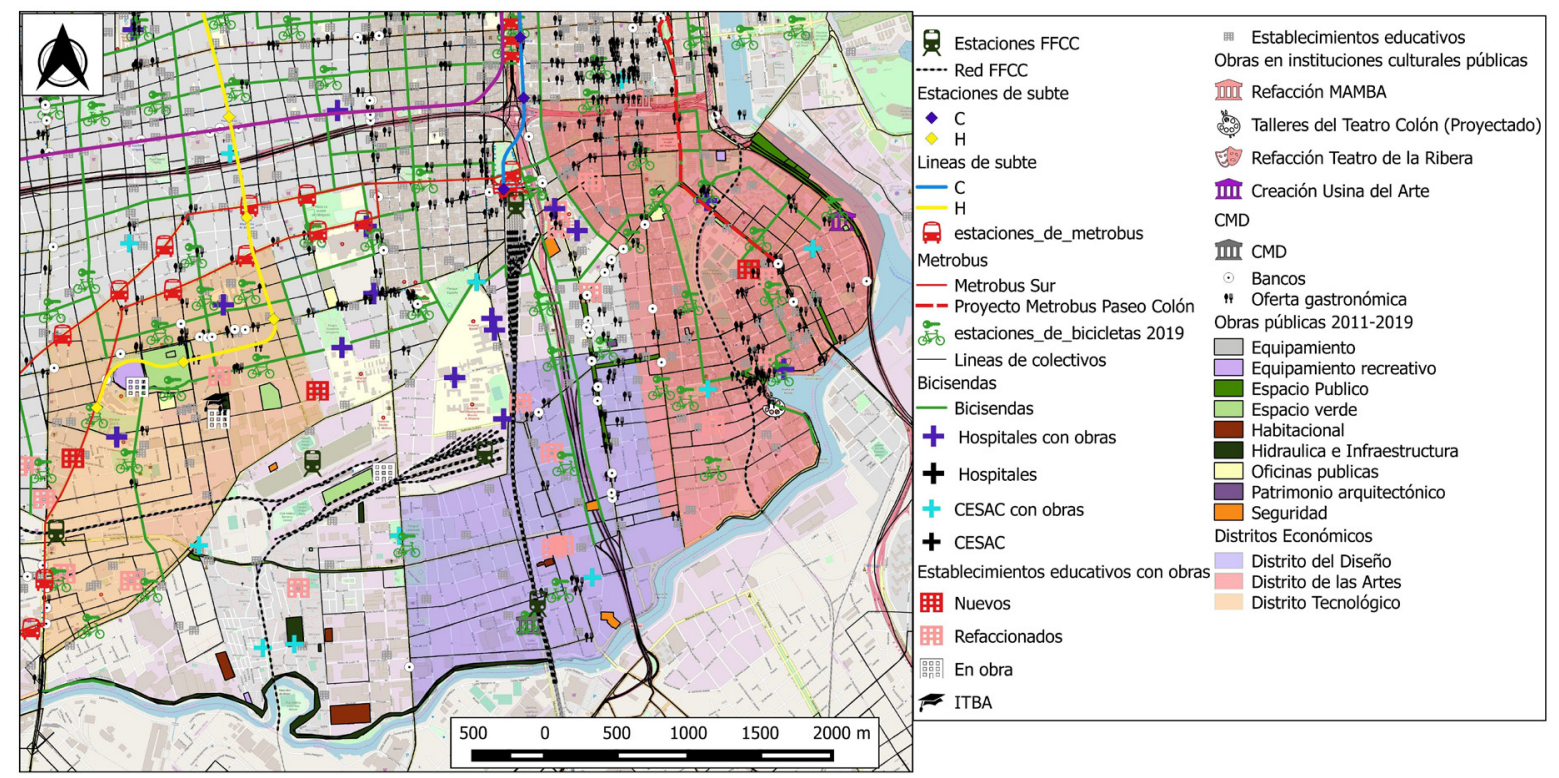

Fuente: Elaboración propia en base a "Buenos Aires Data", s.f.

sobreimprimiéndolas. Esta nueva imagen se apoya en el arte, jerarquizando e hipervisibilizando uno grupo de tradicionales residentes del barrio (los artistas plásticos) y omitiendo otro (los sectores populares). Como apunta Thomasz (2017), una de las herramientas para la construcción de esta nueva imagen artística es recuperar sólo una parte del pasado artístico del barrio -especialmente el legado de Quinquela Martín y del Grupo de La Boca-, destacando el valor estético de sus obras y omitiendo los procesos sociales y políticos que le dieron lugar y con los que las obras dialogaban. Esta subversión del legado quinqueliano (Thomasz, 2017) se conjuga con la atracción de otro tipo de actores vinculados al mercado del arte, y ajenos al barrio, como galerías de arte con proyección internacional, fundaciones de arte vinculadas a empresas privadas y desarrollos inmobiliarios que incorporan proyectos artísticos para obtener los beneficios impositivos. 
Así, en el distrito de las artes, las inversiones públicas se acompañan de una estrategia de comunicación orientada a consolidar la nueva identidad del distrito, subvirtiendo las identidades barriales preexistentes. Mientras que la imagen tradicional "pintoresca" sigue vigente para atraer turismo internacional al enclave turístico de Caminito; la nueva imagen ligada al arte busca atraer visitantes locales (Gonzalez Bracco y Kotschack, 2017), público de "nicho" e inversores ligados al mercado artístico e inmobiliario, ampliando la zona de renovación hacia San Telmo y Puerto Madero. Se construye discursivamente una continuidad sin fisuras entre el "acervo cultural y artístico" de La Boca y el proceso de renovación actual basado en el arte. En este proceso, se omiten los conflictos en torno a esta propuesta y se invisibilizan las problemáticas que golpean a grandes sectores del barrio, entre ellas la emergencia habitacional.

El distrito del diseño apunta a la renovación urbana del sector sudoeste de Barracas, hasta entonces, de escaso interés para el sector privado. Resaltando su valor patrimonial y cultural y utilizando el diseño, el arte urbano y el patrimonio industrial como principales recursos (Hernández, 2017), el distrito se presenta como una nueva propuesta para "recuperar" este sector "olvidado" de Barracas. Aquí, el diseño cumple varias funciones. En primer lugar, retomando las premisas de la economía creativa, se presenta como una solución al deterioro del barrio, proponiéndose como una actividad promotora de empleo y desarrollo que representa una continuidad natural, armónica y sin conflictos con su pasado fabril (Hernández, 2017).

Por otro lado, el diseño se presenta como una disciplina adecuada para intervenir y "embellecer" el espacio urbano, dando color a esta zona "gris" de la ciudad (Hernández, 2015, 2017). A la vez, esta intervención en el espacio público es convertida en un atractivo, tanto para turistas como para potenciales inversores. Al igual que en el distrito de las artes, la generación de eventos y recorridos temáticos en esta zona de la ciudad, como Casa FOA, Mirar Barracas (2014), La Noche de los Museos, Open House (2015), también se orienta en este sentido.

A partir de una serie de eventos vinculados al campo de la arquitectura y la decoración, inversores inmobiliarios y organismos del GCBA comienzan a movilizar la categoría de patrimonio industrial. Por un lado, esta categoría sirve para legitimar los proyectos inmobiliarios de refuncionalización, unificando el interés de desarrolladores que funcionan en la lógica de la ganancia con la demanda de las organizaciones vecinales que hacen uso del patrimonio para evitar la densificación y verticalización del barrio (Hernández, 2015, 2017). De manera complementaria, la categoría de patrimonio industrial construye una imagen distintiva y singular del barrio que, apoyándose en determinados edificios emblemáticos, 
refuerza la identidad "industrial" de Barracas como un valor que mejora sus condiciones de atractividad. Así, en el proceso de renovación de Barracas, el patrimonio industrial opera como doble recurso para la valorización de la zona: como nuevo producto inmobiliario y, a la vez, como atractivo turístico.

La construcción de una nueva imagen requiere, al mismo tiempo, de invisibilizar algunos rasgos y actores de las áreas sujetas a renovación. En este caso, se oculta la historia de lucha de los trabajadores de las fábricas del barrio (Hernández, 2017) y la problemática habitacional que afecta a los residentes de las villas y asentamientos de Barracas. Asimismo, se produce un vaciamiento simbólico que presenta como "vacíos" algunos espacios que no lo están, pero que contienen actividades "no deseadas", como el caso de los hospitales psicoasistenciales y los galpones linderos al Riachuelo que concentran actividades logísticas (Gonzalez Redondo, 2020).

El mayor avance del distrito tecnológico, por una cuestión temporal y por la mayor facilidad para atraer empresas, incide en que la renovación no requiera ser apuntalada por políticas de activación patrimonial y turística. El hecho de que aquí los potenciales visitantesconsumidores sean los propios trabajadores de las empresas TIC, con buenos salarios y capacidad de consumo, hace que los factores artísticos, patrimoniales y turísticos queden relegados a un segundo plano. Esto es visible, por ejemplo, en los eventos realizados en el marco del distrito orientados exclusivamente a la industria TIC. Otro rasgo particular es el tratamiento que se le da a los inmuebles industriales que, al igual que en Barracas, predominan en el área sur del distrito. En este distrito no se moviliza la categoría de patrimonio industrial. Por lo contrario, estos inmuebles en general son ofrecidos como terrenos para su redesarrollo, borrando el pasado industrial y proponiendo un presente moderno, con edificios de oficinas de alta gama, en sintonía con la construcción argumentativa que se hace del área y de su proceso de renovación.

Así, el proceso de renovación en el área del DT implicó la construcción de un nuevo imaginario, vinculado a la innovación, la tecnología, el talento y la creatividad -todos significantes asociados a la actividad que desarrollan las empresas TIC- que convive con atributos propios de la centralidad (específicamente su accesibilidad) y de la fisonomía barrial de esta zona, como sus casas bajas y sus espacios verdes reacondicionados. Otro aspecto de esta narrativa es el proceso de colonización de esta zona considerada "tierra de nadie". Son recurrentes los relatos periodísticos que tensionan el pasado inseguro y marginal del barrio con su presente tranquilo y "lleno de vida", asociado a la "llegada" de empresas TIC, de jóvenes "talentosos y creativos", de "inversiones comprometidas" que vieron el potencial del distrito y "apostaron" junto con el GCBA a la 
renovación del área (Castiglione, 2018; "10 años del Distrito Tecnológico", 2018; Lopardo, 2018; Rotnitzky, 2018). Al igual que sucede en los otros distritos, se construye un protagonista de la renovación que en este caso gira en torno a la presencia sobredimensionada de la figura de los empresarios y empleados jóvenes, creativos y talentosos de la industria TIC.

El proceso de renovación refuerza la segmentación previa del área, con una mayor concentración de empresas, desarrollos inmobiliarios y nuevos comercios en la zona norte del distrito y una mayor dispersión hacia el sur (Centro de Estudios para el Desarrollo Económico Metropolitano, 2011, 2013, 2014; Goicoechea, 2016). Las sucesivas intervenciones públicas y privadas han intentado extender esa frontera material y simbólica hacia el sur, con éxito creciente a lo largo del tiempo; sin embargo, esta frontera todavía persiste. Estos esfuerzos también se observan en el entramado discursivo sobre el distrito que, mientras hipervisibiliza las transformaciones y sus nuevos visitantes y habitantes, omite las problemáticas sociales y económicas que golpean a la población vulnerable de la zona. De este modo, los hogares y centros de día para población en situación de calle, la atención a jóvenes con consumo problemático de sustancias y la problemática habitacional de la villa 21-24 (que limita al sur del distrito) no forman parte de la agenda del gobierno local ni de los relatos sobre el nuevo barrio.

\section{Conclusiones}

En este artículo desplegamos una estrategia metodológica combinada para analizar las múltiples intervenciones públicas orientadas a crear el "clima de negocios" en las áreas de los distritos. En primer lugar, la triangulación de fuentes permitió cuantificar la inversión pública y así evaluar el papel del Estado en la implementación de esta política. Advertimos que -contrariamente a lo que se sostiene en el discurso oficial-, el gobierno local desplegó una gran (aunque desigual) inversión que no fue acompañada con la misma magnitud por el sector privado.

En segundo lugar, el análisis discursivo resultó productivo en dos sentidos. Primero, permitió advertir cómo se perfilan los roles de mediador y facilitador del Estado local en esta política y cómo éstos son percibidos y valorados por los actores empresariales. Segundo, contribuyó a identificar las narrativas que se construyen para legitimar las intervenciones en curso (Franquesa, 2007) y, a la vez, resemantizar (Thomasz, 2017) los barrios que son objeto de intervención. Tanto en la construcción de estas narrativas, como en las modalidades de intervención de los actores estatales y en las intervenciones desplegadas en el territorio, advertimos un papel preponderante del Estado local en la creación del "clima de negocios". 
Por último, el mapeo de las intervenciones públicas permitió visualizar cómo se distribuyen desigualmente las intervenciones en el territorio, tanto entre como intra distritos. Este análisis echó luz sobre la articulación de esta política con procesos e intervenciones preexistentes, enfatizando el carácter del espacio como sistema de objetos (Santos, 2000) que modula la acción transformadora de la política urbana. Las intervenciones públicas englobadas en la política de distritos tienden a reforzar las desigualdades preexistentes y persistentes. Mientras que algunos sectores de los distritos muestran signos de transformación; otros permanecen en el olvido. Los diferentes ritmos de los procesos económicos y territoriales producen una mezcla compleja entre continuidad y cambio (Brenner, 2004; Cuervo González, 2006, 2017), advirtiendo que los distritos no sólo se montan sobre proyectos y objetos heredados, sino también sobre institucionalidades y configuraciones de poder heredadas (Theodore et al., 2009).

Estos hallazgos enfatizan la importancia de las dimensiones espacial y temporal en el estudio de los procesos y políticas urbanas, sobre todo para dar cuenta de sus continuidades y persistencias. Asimismo, resaltan la capacidad de agencia de los actores y de sus trayectorias en el devenir de una política urbana, dado que, como se desprende de nuestro análisis, algunas de sus características novedosas obedecen a la impronta de los sujetos que habitan y dan vida a esta politica (Bohoslavsky y Soprano, 2008). La perspectiva de la sociología política, junto con el abordaje discursivo fueron especialmente útiles para detectar estas particularidades. La inclusión de estas herramientas, que invitan a pensar al Estado en su heterogeneidad, permite complejizar el análisis de políticas urbanas. Muchas de las corrientes que abordaron críticamente este fenómeno se sitúan en un plano de abstracción más general y fueron desarrolladas en el norte global, sin ajustarse a las particularidades de nuestros territorios. Tal es el caso de la categoría empresarialismo urbano (Harvey, 1989) que, como vimos, no define acabadamente el tipo de gestión urbana que advertimos en la política de distritos. Algo similar sucede con el concepto de neoliberalismo realmente existente (Theodore et al., 2009) que, si bien sirve como un marco general para pensar cómo el neoliberalismo se produce de diversas formas y con efectos diferentes en cada lugar, no brinda herramientas para el análisis en un nivel más sustantivo. De ahí que el análisis ensayado en este artículo resulte útil para llenar de contenido estas categorías más abstractas y a la vez reflexionar sobre los procesos de gobernanza urbana.

En esta política, losesfuerzos públicos orientados a crear el "clima de negocios" y a la atracción de inversiones a los distritos tuvieron una respuesta limitada por parte del sector privado. Si bien prima un discurso que sostiene que el Estado "no pone un peso" y que el privado "apuesta" 
a los distritos, advertimos que éste último se limita en general a aprovechar los incentivos públicos asumiendo muy pocos riesgos. Esto nos lleva a enfatizar la importancia de analizar en detalle las respuestas que produce el sector privado ante los incentivos públicos. Esta dimensión analítica -que, por restricciones de espacio, queda pendiente para futuros trabajoscontribuirá a seguir cuestionando el potencial de las "sinergias público-privadas" y de los procesos de gobernanza urbana para propiciar el desarrollo de "áreas deterioradas".

\section{Referencias bibliográficas}

Aguilar, L. (2016). Gobernanza y gestión pública. Ciudad de México: Fondo de Cultura Económica.

Angenot, M. (2010). El discurso social: problemática de conjunto. En El discurso social: los límites históricos de lo pensable y lo decible (pp. 21-49). México: Siglo XXI

Arqueros, S. (2019). La política de desarrollo de la Comuna 8 de la ciudad de Buenos Aires (1996-2015). Quid 16, (11), 331-337.

Arqueros Mejica, S. y Gonzalez Redondo, C. (2017). La política de distritos del sur de Buenos Aires: una mirada en perspectiva. Quid 16, (7), 7-29.

BA Obras. (s.f.). Buenos Aires Ciudad. Recuperado de https://data.buenosaires.gob.ar/dataset/ba-obras

Becattini, G. (2002). Del distrito industrial marshalliano a la "teoría del distrito" contemporáneo. Una breve reconstrucción crítica. Investigaciones Regionales, (1), 9-32.
Bohoslavsky, E. y Soprano, G. (2010). Una evaluación y propuestas para el estudio del Estado en Argentina. En Un Estado con rostro humano. Funcionarios e instituciones estatales en Argentina (de 1880 a la actualidad). Buenos Aires: Prometeo Libros.

Bracco, M. G. y Kotschack, L. (2017). El espacio turístico, entre el enclave y el derrame: estudio en dos barrios de Buenos Aires. Cuadernos de Geografía: Revista Colombiana de Geografía, 26(2), 373-397. https://doi.org/10.15446/rcdg.v26n2.59182

Brenner, N. (2004). New state spaces. Urban governances and the rescaling of statehood. New York: Oxford University Press.

Buenos Aires Data. (s.f.). Buenos Aires Ciudad. Recuperado de https://data.buenosaires.gob.ar/

Castiglione, J. (2018). Polo Tecnológico: atraídas por ventajas impositivas, ya hay 314 empresas en el "Silicon Valley" argentino. IproUP. Recuperado de https://www.iproup.com/innovacion/758-parque-patricios-distrito-tecnol\%C3\%B3gico-Distrito-Tecnologico-con-Mercado-Libre-ya-hay-314-empres

Centro de Estudios para el Desarrollo Económico Metropolitano. (2011). La apuesta del Distrito Tecnológico en el sur de la Ciudad. Un análisis de la estructura de ocupación de la zona. Buenos Aires: Autor.

Centro de Estudios para el Desarrollo Económico Metropolitano. (2013). La apuesta del Distrito Tecnológico en el sur de la Ciudad. Un análisis de la estructura de ocupación de la zona. Segundo informe de avance. Buenos Aires: Autor. 
Centro de Estudios para el Desarrollo Económico Metropolitano. (2014). La apuesta del Distrito Tecnológico en el sur de la Ciudad. Un análisis de la estructura de ocupación de la zona. Tercer informe de avance. Buenos Aires: Autor.

CEPAL. (2018). Plan de acción regional para la implementación de la nueva agenda urbana en América Latina y el Caribe (2016-2036). Santiago: Autor.

Cities in transition: World Bank urban and local government strategy. (2000). Washington, D.C.: The World Bank.

Cuervo González, L. M. (2006). Globalización y territorio. Santiago de Chile: ILPES-CEPAL.

Cuervo González, L. M. (2017). Ciudad y territorio en América Latina. Bases para una teoría multicéntrica, heterodoxa y pluralista. Santiago de Chile: CEPAL.

Dávila, J. (2014). Urban fragmentation, 'good governance' and the emergence of the competitive city. En S. Parnell y S. Oldfield (Coords.) The Routlegde handbook on cities of the global South. New York: Routledge.

Di Virgilio, M. y Guevara, T. (2014). Gentrificación liderada por el Estado y empresarialismo urbano en la Ciudad Autónoma de Buenos Aires. Estudios Sociales Contemporáneos, (11), 12-23.

Di Virgilio, M. y Rodríguez, C. (2016). De políticas públicas, escalas y territorios: La ciudad como objeto de intervención. En C. Rodríguez y M. Di Virgilio (Comps.), Territorio, políticas habitacionales y transformaciones urbanas. Buenos Aires: Espacio Editorial.
10 años del Distrito Tecnológico: crecimiento y evolución. (2018). Buenos Aires Ciudad. Recuperado de https://www.buenosaires.gob.ar/ economiayfinanzas/noticias/10-anos-del-distrito-tecnologico-crecimiento-y-evolucion

Fernández, V. R., Amin A. y Vigil, J. I. (Comps.). (2008) Repensando el desarrollo regional. Contribuciones globales para una estrategia latinoamericana. Buenos Aires: Miño y Dávila.

Franquesa, J. (2007). Vaciar y llenar, o la lógica espacial de la neoliberalización. Reis: Revista Española de Investigaciones Sociologicas, (118), 123-150. https://doi.org/10.2307/40184799

Gené, M. (2011). En torno a los profesionales de la política. Trayectorias, prácticas y destrezas en el ejercicio del poder político desde el estado. Revista Perspectivas de Políticas Públicas, 1(1), 85-107. https://doi.org/10.18294/ rppp. 2011.645

Giorgi, G. I. (2014). Ministros y ministerios de la Nación argentina: un aporte prosopográfico para el estudio del gabinete nacional (18542011). Apuntes. Revista de ciencias sociales, 41(74), 103-139. https://doi.org/10.21678/ apuntes. 74.704

Goicoechea, M. (2018). Distritos creativos en el sur de la ciudad de Buenos Aires (2008-2015). Renovación urbana y nuevas lógicas de segregación. Quid 16, (9), 224-227.

Gonzalez Redondo, C. (2017). "Un porteño más”. Los discursos sobre el turismo alternativo en la ciudad de Buenos Aires. III Jornadas Internacionales de Antropología del Conflicto Urbano, Buenos Aires, Argentina. 
Gonzalez Redondo, C. (2018). Del distrito gubernamental a la nueva sede de gobierno. Disputas en torno al espacio urbano en la ciudad de Buenos Aires. (Tesis Magister en Políticas Ambientales y Territoriales, sin publicar). Universidad de Buenos Aires, Argentina.

Gonzalez Redondo, C. (2019). La política de distritos en el sur de la ciudad de Buenos Aires. Modelos internacionales, actores locales y territorio (20082019). (Tesis doctoral, sin publicar). Universidad de Buenos Aires, Argentina.

Gonzalez Redondo, C. (2020). El vaciamiento que antecede la renovación. Reflexiones a partir del proyecto del distrito gubernamental en el sur de la ciudad de Buenos Aires. Territorios, (42). https://doi.org/10.12804/revistas.urosario.edu.co/territorios/a.7228

Harvey, D. (1989). From managerialism to entrepreneurialism: the transformation in urban governance in late capitalism. Geografiska Annaler. Series B, Human Geography, 71(1), 3-17. https://doi.org/10.2307/490503

Hernández, S. (2015). Casa FOA y la emergencia del patrimonio industrial como valor especifico del barrio de Barracas. XI Jornadas de Sociología. Buenos Aires, Facultad de Ciencias Sociales, Universidad de Buenos Aires.

Hernández, S. (2017). El rol del Centro Metropolitano de Diseño en el proceso de patrimonialización de Barracas (Ciudad de Buenos Aires ). Quid 16, (7), 94-119.

Leitner, H. (1990). Cities in pursuit of economic growth. Political Geography Quarterly, 9(2), 146-170. https://doi.org/10.1016/0260-9827(90)90016-4
Lopardo, L. (2018). Todo lo que hay que saber antes de instalarse en el Distrito Tecnológico. La Nación. Recuperado de https://www.lanacion.com.ar/ propiedades/inmuebles-comerciales/todo-lo-hay-saber-antes-instalarse-distrito-nid2167713

Lopes de Souza, J. (1995). O territorio: sobre espaco e poder, autonomia e desenvolvimiento. En I. Elias de Castro, P. C. da Costa Gomes, y R. Lobato Correa (Orgs.), Geografia: conceitos e temas. Río de Janeiro: Bertrand.

Marcús, J. y Vázquez, D. (2017). Vaciar, llenar, resistir. Conflictos y tensiones en la producción de 'vacíos urbanos'. En J. Marcús (Coord.), Ciudad viva. Disputas por la producción sociocultural del espacio urbano en la Ciudad de Buenos Aires (pp. 33-66). Buenos Aires: Teseo.

Marques, E. (2013). Government, political actors and governance in urban policies in Brazil and São Paulo: concepts for a future research agenda. Brazilian Political Science Review, 7(3), 8-35. https://doi.org/10.1590/ $\underline{\text { s1981-38212013000300001 }}$

Marques, E. (2019). Notas sobre redes, Estado e políticas públicas. Cadernos de Saúde Pública, 35(sup. 2), 1-11. https://doi.org/10.1590/0102-311x00002318

Mattos, C. d. (2008). Globalización, negocios inmobiliarios y mercantilización del desarrollo urbano. En M. Córdova (Coord.), Lo urbano en su complejidad: una lectura desde América Latina (pp. 35-62). Quito: Flacso.

Menazzi, L. y Jajamovich, G. (2019). Saberes urbanos. Buenos Aires. 
Nueva agenda urbana. (2017). Habitat III. Recuperado de http://habitat3.org/wp-content/uploads/ NUA-Spanish.pdf

OECD. (2000). The reform of metropolitan governance. Washington: Autor.

Pírez, P. (2016). Buenos Aires: la orientación neoliberal de la urbanización metropolitana. Sociologias, 18(42), 90-118.

https://doi.org/10.1590/15174522-018004204

Porter, M. (1995). The competitive advantage of the inner city. Harvard Bussiness Review, 73(3), $55-71$.

Porter, M. (1998). Clusters and the new economic competition. Harvard Business Review, 76(6), 77-90.

Rodríguez, M. C. y Di Virgilio, M. (2011). Coordenadas para el análisis de las políticas urbanas: un enfoque territorial. En M. C. Rodriguez y M. Di Virgilio (Comps), Caleidoscopio de las politicas territoriales. Un rompecabezas para armar. Buenos Aires: Prometeo.

Rotnitzky, N. (2018). Cómo el distrito tecnológico transformó Parque Patricios. La Nación. Recuperado de https://www.lanacion.com.ar/sociedad/ el-distrito-tecnologico-transformo-parque-patricios-cumple-nid2192412

Salmerón Escobar, F. (2017). Un lugar en la mesa global. Los gobiernos locales como tomadores de decisiones en la agenda mundial. Ciudad de México: Gobierno de la Ciudad de México.

Santos, M. (2000). La naturaleza del espacio: técnica y tiempo, razón y emoción. Barcelona: Ariel.
Shore, C. (2010). La antropología y el studio de la política pública: reflexiones sobre la "formulación” de las políticas públicas. Antípoda, (10), 2149. https://doi.org/10.7440/antipoda10.2010.03

Stanchieri, M. L. y Aricó, G. (2013). La trampa urbanística de los "vacíos urbanos": casos etnográficos en Barcelona. X Jornadas de Sociología. Buenos Aires, Argentina.

Theodore, N., Peck, J., y Brenner, N. (2009). Urbanismo neoliberal: la ciudad y el imperio de los mercados. Temas Sociales, (66).

Thomasz, A. G. (2017). Etnografía de un proceso de resemantización simbólico: del barrio de La Boca a Distrito de las Artes. Quid 16, (7), 67-93.

Vainer, C. (2000). Pátria, empresa e mercadoria. Notas sobre a estratégia discursiva do Planejamento Estratégico Urbano. En O. Arantes, C. Vainer y E. Maricato, A cidade do pensamento único: desmanchando consensos (pp. 75-103). Río de Janeiro: Vozes.

Vommaro, G. (2017). La larga marcha de Cambiemos. La construcción silenciosa de un proyecto de poder. Buenos Aires: Siglo XXI.

Vommaro, G. y Gené, M. (Comps.). (2017). La vida social del mundo político. Investigaciones recientes en sociología política. Los Polvorines: UNGS.

Zurbriggen, C. (2011). Gobernanza: una mirada desde América Latina. Perfiles Latinoamericanos, 19(38), 39-64. 


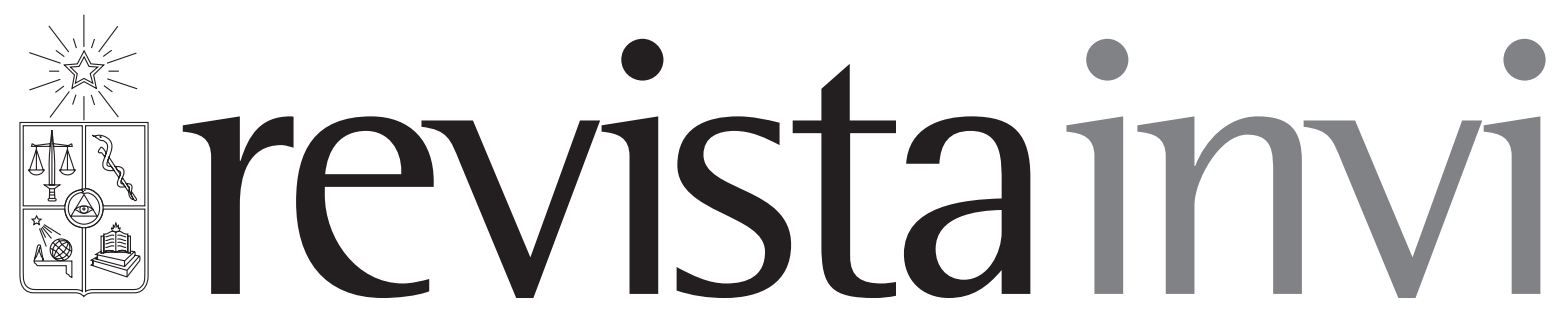

Revista INVI es una publicación periódica, editada por el Instituto de la Vivienda de la Facultad de Arquitectura y Urbanismo de la Universidad de Chile, creada en 1986 con el nombre de Boletín INVI. Es una revista académica con cobertura internacional que difunde los avances en el conocimiento sobre la vivienda, el hábitat residencial, los modos de vida y los estudios territoriales. Revista INVI publica contribuciones originales en español, inglés y portugués, privilegiando aquellas que proponen enfoques inter y multidisciplinares y que son resultado de investigaciones con financiamiento y patrocinio institucional. Se busca, con ello, contribuir al desarrollo del conocimiento científico sobre la vivienda, el hábitat y el territorio y aportar al debate público con publicaciones del más alto nivel académico.

Director: Dr. Ricardo Tapia Zarricueta, Universidad de Chile, Chile.

Editor: Dr. Luis Campos Medina, Universidad de Chile, Chile.

Editor asistente: Dr. Walter Imilan, Universidad de Chile, Chile.

Coeditora: Srta. Sandra Rivera, Universidad de Chile, Chile.

\section{COMITÉ EDITORIAL:}

Dr. Victor Delgadillo, Universidad Autónoma de la Ciudad de México, México.

Dra. María Mercedes Di Virgilio, CONICET/ IIGG, Universidad de Buenos Aires, Argentina.

Dra. Irene Molina, Uppsala Universitet, Suecia.

Dr. Gonzalo Lautaro Ojeda Ledesma, Universidad de Valparaíso, Chile.

Dra. Suzana Pasternak, Universidade de São Paulo, Brasil.

Dr. Javier Ruiz Sánchez, Universidad Politécnica de Madrid, España.

Dra. Elke Schlack Fuhrmann, Pontificia Universidad Católica de Chile, Chile.

Dr. Carlos Alberto Torres Tovar, Universidad Nacional de Colombia, Colombia.

Sitio web: http://www.revistainvi.uchile.cl/

Correo electrónico: revistainvi@uchilefau.cl

Licencia de este artículo: Creative Commons Atribución-Compartirlgual 4.0 Internacional (CC BY-SA 4.0) 